\title{
6. Herbsttagung der Deutschen Gesellschaft für Allgemein- \& Viszeralchirurgie - eine Nachschau
}

\section{6th Autumn Meeting of the German Society for General and Visceral Surgery - Selected Topics}

Vom 19.-22. September 2012 fand in Hamburg zum 6. Mal die Herbsttagung der Deutschen Gesellschaft für Allgemein- \& Viszeralchirurgie statt. Nach 2009 war die Hansestadt schon zum 2. Mal Austragungsort der gemeinsamen Tagung der 2 wichtigsten viszeralmedizinischen wissenschaftlichen Fachgesellschaften, der Deutschen Gesellschaft für Verdauungs- und Stoffwechselkrankheiten mit der Sektion für gastroenterologische Endoskopie (DGVS) und der Deutschen Gesellschaft für Allgemein- und Viszeralchirurgie (DGAV) mit ihren Arbeitsgemeinschaften.

Der gemeinsame Anspruch der Tagungspräsidenten Prof. Dr. Peter Layer (DGVS, Hamburg) und Prof. Dr. Stefan Post (DGAV, Mannheim) war es, einen Kongress zu gestalten, der dem intensiven wissenschaftlichen Austausch und der Vermittlung aktueller Forschungsergebnisse genauso diente wie der Vermittlung wichtiger praxisrelevanter Fortschritte. Auch wurde die Werbung um den qualifiziertesten Nachwuchs für die Viszeralmedizin nicht nur gefordert, sondern in einem attraktiven und bisher nicht dagewesenen Umfang in den Kongress integriert.

Einen Akzent setzte der Präsident der DGAV Prof. Stefan Post schon im Rahmen der Eröffnungssitzung am Mittwochnachmittag. Im Hinblick auf den Nachwuchsund Ärztemangel forderte er, nicht in das bekannte Lamento einzufallen, sondern stellte pointiert fest, dass die Ärztedichte in Deutschland im internationalen Vergleich hoch sei, die Deutschen deutlich häufiger als ihre europäischen Nachbarn den Arzt aufsuchten und es ein hohes Maß an Redundanzen zwischen dem ambulanten und stationären Sektor gäbe. Seiner Meinung nach liegt die Lösung der aktuellen Problematik nicht in „noch mehr Ärzten“, sondern im sinnvollen Ressourceneinsatz und dem Aufbrechen nicht mehr zeitgemäßer Versorgungsstrukturen.
Ein Höhepunkt des 1. Kongresstags am Mittwoch, der traditionell von den gut besuchten und inhaltlich hochkarätigen Postgraduiertenkursen geprägt war, war die Verleihung der Walter-Kausch-Medaille an Keith D. Lillemoe, den Direktor des Department of Surgery des Massachusetts General Hospital und Professor an der Harvard Medical School, Boston, USA. Professor Lillemoe, der seine akademische und chirurgische Ausbildung an der Johns Hopkins Universität Baltimore erhielt, war von 2003 bis 2011 Leiter der Chirurgischen Klinik an der Indiana Universität in Indianapolis, bevor er an die Harvard Medical School berufen wurde. Durch seine umfangreiche wissenschaftliche und klinische Expertise im Bereich benigner und maligner Pankreasund Gallenwegserkrankungen steht Professor Lillemoe als 1. US-amerikanischer Träger der Walter-Kausch-Medaille in einer Linie mit den vorhergehenden Preisträgern Prof. Ihse, Lund, Schweden, Prof. Trede, Mannheim, und Prof. Beger, Ulm. Und so würdigte Prof. Lillemoe in seinen Dankesworten auch die besonderen Verdienste von Walter Kausch, der bereits 1909 und damit 25 Jahre vor Allen O. Whipple, die 1. partielle Duodenopankreatektomie durchführte.

Die klassischen Kongresstage Donnerstag und Freitag standen ganz im Zeichen wissenschaftlicher Vorträge. Hierbei reichte das angebotene Spektrum von den wissenschaftlichen Grundlagen bis zu aktuellen Leitlinienempfehlungen.

Auch schon in guter Tradition wurden die 11 Arbeitsgemeinschaften der DGAV in die Kongressplanung eingebunden und richteten eigenständig AG-Sitzungen aus, in denen die aktuellen Fragen und Techniken des jeweiligen Schwerpunkts diskutiert wurden. Sehr positiv wurde aufgenommen, dass die Kongressteilnehmer mit maximal 3 parallel laufenden chirurgischen Hauptsitzungen mit jeweils unterschiedlichen Themenschwerpunkten die Möglichkeit hatten, ihr individuelles Kongressprogramm entsprechend der je- weiligen Interessen zu gestalten. Hierbei war auch die kostenlose Kongress-App für Smartphones eine viel genutzte Hilfe. Besonders großes Interesse fanden die Vorträge mit ganz praktischem Bezug wie die Sitzung: „Chirurgie des Häufigen: Was ist wirklich evidenzbasiert?“ oder die Sitzungen mit aktiver Teilnahme aller Zuhörer. Die 2 DGAV-TED-Sitzungen zum Themenschwerpunkt „Komplikationsmanagement" übertrafen nicht nur das Fassungsvermögen der Säle bei Weitem. Auch inhaltlich waren die Fallpräsentationen vor dem hochkarätigen Expertengremium mit der Möglichkeit der interaktiven Teilnahme eine Bereicherung für alle Zuhörer - vom Medizinstudenten bis zum emeritierten Ordinarius -, was die regen Diskussionen bewiesen.

Ohnehin stand die Nachwuchsförderung ganz im Fokus der diesjährigen Viszeralmedizin. Erstmalig wurde ein Tutorenprogramm für Medizinstudenten der klinischen Semester angeboten. In Begleitung eines erfahren Fach- oder Oberarztes besuchten die Studenten in Kleingruppen ausgewählte Sitzungen, die gezielt vor- und nachbereitet wurden. Durch eine bunte Mischung verschiedener gastroenterologisch-endoskopischer und viszeralchirurgischer Sitzungsthemen wurde so den Studenten die Vielfalt der Fächer nähergebracht.

Als weiterer Aspekt der Nachwuchsförderung konnten auch die neu geschaffenen Kurzvortragssitzungen gesehen werden. Anstelle der konventionellen Posterbegehungen wurden erstmalig alle angenommenen Abstracts als kompakte Vorträge in eigenen Sitzungen präsentiert. Von Donnerstag 8:30 Uhr bis Freitag 19:00 Uhr tauschte sich der wissenschaftliche Nachwuchs in 11 Kurzvortragssitzungen quer durch alle viszeralchirurgischen Themenbereiche aus - von anspruchsvollen, onkologischen Themen bis zu klinisch-herausfordernden Kasuistiken. Um den besonderen Stellenwert dieser Sitzungen noch weiter zu betonen, hatte der DGAVVorstand für jede der 11 Sitzungen einen mit 300 Euro dotierten Vortragspreis ausgelobt.

Im Zentrum standen auch in diesem Jahr die interdisziplinären Sitzungen. Hierbei wurden klinisch relevante Themen wie die Obstipation genauso beleuchtet wie die großen Themen der Viszeralmedizin. In kompakten Update-Sitzungen konnten sich die Teilnehmer über die aktuellsten wissenschaftlichen und klinischen Entwicklungen zu Themen wie den Malignomen des Gastrointestinaltrakts bis hin zu 
den chronisch-entzündlichen Darmerkrankungen austauschen.

Neben den wissenschaftlichen, viszeralmedizinischen Schwerpunkten der Herbsttagung wurden aber auch alternative Themenfelder besetzt. Besonders reges Interesse weckte die Sitzung „Der zerbrochene Krug' - Recht in der Viszeralmedizin“, hier beleuchteten die Referenten Themen wie Aufklärungsfehler, Delegation ärztlicher Tätigkeiten und Hygienemängel mit ihren rechtlichen Folgen.

Neben den Vortragspreisen wurde unter allen 173 eingereichten chirurgischen Abstracts zum 5. Mal der mit $€ 2000$ dotierte Carl-Langenbuch-Preis für den inhaltlich, formal und sprachlich besten freien Vortrag vergeben. Hier konnte Frau Dr. Petra Kühn, Oberärztin an der Chirurgischen Klinik des Universitätsklinikums/ Knappschaftskrankenhauses Bochum mit ihrem Vortrag „Arbeitsbedingungen in der Chirurgie - was wir besser machen können“ überzeugen.

Der Samstag stand dann ganz im Zeichen der Interdisziplinarität und der Übergabe des Staffelstabs an den kommenden Präsidenten der DGAV, Professor Dr. Matthias Anthuber aus Augsburg. In der Ab- schlusssitzung „Highlights aus der Viszeralmedizin 2012“ betonten die künftigen Kongresspräsidenten der Viszeralmedizin 2013 in Nürnberg ihre Höhepunkte des vergangenen Jahres. Für Professor Anthuber war dies insbesondere die Leberchirurgie, die unter anderem mit dem In-situ-Splitt-Verfahren als innovativem Therapieansatz die Grenzen der Resezierbarkeit von Leberprozessen neu zu definieren scheint. Ebenso spannend fand er die Frage nach dem richtigen Zeitpunkt einer Cholezystektomie bei akuter Cholezystitis. Mit Verweis auf die deutsche ACDCStudie, der größten prospektiv-randomisierten Studie zu diesem Thema, zeigte er, dass es nun endlich klare Evidenz für eine frühe Cholezystektomie gibt. Rückblick und Ausschau zugleich waren auch Professor Anthubers Ausführungen zum Stellenwert der laparoskopischen kolorektalen Chirurgie. Hierzu betonte er, dass sich die laparoskopischen Techniken auch in der Chirurgie kolorektaler Malignome langsam etablieren. Er vermisse jedoch diesbezügliche Daten über die Situation in Deutschland.

Nicht weniger relevant, aber mit einem Schmunzeln vorgetragen und so auch vom Auditorium aufgenommen, waren seine abschließenden Worte zur Ausbildung des chirurgischen Nachwuchses, vom Studenten zum „Homo chirurgicus“. Den Schlusspunkt dieser Highlight-Sitzung aber setzte ein Internist. Professor Gerken, der DGVS-Kongresspräsident 2013, stellte klar, die Viszeralmedizin 2012 selbst war ein Highlight.

\section{Interessenkonflikt: Nein}

T. Hasenberg, J. Jonescheit, M. Niedergethmann, S. Post

Chirurgische Klinik, Universitätsklinikum Mannheim, Mannheim, Deutschland

Bibliografie

DOI http://dx.doi.org/10.1055/s-1328345

Online-publiziert: 03.07.2013

Zentralbl Chir 2013; 138: 17-18

(c) Georg Thieme Verlag KG Stuttgart - New York . ISSN 0044-409X

Korrespondenzadresse

Dr. Till Hasenberg

Chirurgische Klinik

Universitätsklinikum Mannheim

Theodor-Kutzer-Ufer 1-3

68167 Mannheim

Deutschland

Tel.: 0621/383-2225

Fax: 0621/383-3809

till.hasenberg@krupp-krankenhaus.de 